\title{
A serogrouping scheme for the study of the epidemiology of Bacteroides fragilis
}

\author{
K. M. ELHAG and A. SENTHILSELVAN*
}

\section{Departments of Microbiology and ${ }^{*}$ Community Medicine, Faculty of Medicine, The University of Kuwait, PO Box 24923, Safat, Kuwait 13110}

\begin{abstract}
Summary. To study the hospital epidemiology of Bacteroides fragilis, 343 isolates from infected hospitalised patients (112), infected out-patients (102), and from the faeces of uninfected hospitalised patients (47) and normal subjects in the community (82), were examined by an immunofluorescence technique. In tests with antisera against strains of 20 distinct serotypes of the fragilis group of Bacteroides, $271(79 \%)$ strains were typable. Similarity between strains was estimated by the Jaccard similarity measure and strains were then serogrouped by cluster analysis; $88.1 \%$ of hospital strains were typable but only $71.2 \%$ of community strains $(\mathrm{p}<0.001)$. Three serogroups were prevalent among both faecal and infection isolates of hospital strains $(\mathrm{p}<0.01)$. However, no particular serogroup was prevalent among community strains and no difference was found in the distribution of serogroups between strains from faeces and those from infected lesions. One serogroup showed a significant increase $(\mathrm{p}<$ 0.05 ) within the period of study. These findings suggest that strains of $B$. fragilis infecting hospitalised patients may be acquired in hospital and that they may spread to other patients.
\end{abstract}

\section{Introduction}

Bacteroides fragilis is the anaerobe most commonly associated with infection (Moore et al., 1969; Gorbach and Bartlett, 1974; Elhag et al., in press). Although it is the most frequently encountered pathogen of the fragilis group of Bacteroides, it represents only $0.5 \%$ of the bowel microflora and is substantially outnumbered by other members of the group (Moore, 1977). It is now well established that the virulence of $B$. fragilis is associated with cell-surface components such as capsular polysaccharide, outer membrane and lipopolysaccharide (Kasper and Onderdonk, 1982; Jones and Gemmell, 1986; Namavar et al., 1987). Serotyping of $B$. fragilis based on surface structures has shown a wide variety of antigens within this species (Lambe and Moroz, 1976; Elhag et al., 1977; Elhag and Tabaqchali, 1978a; Tabaqchali et al., 1982). Some serotypes were prevalent among strains causing infection (Romond et al., 1972; Elhag and Tabaqchali, 1978b) and different serotypes were prevalent in different hospitals (Elhag and Tabaqchali, $1978 b$ ). This variation in the distribution of $B$. fragilis serotypes possibly indicates spread of these organisms between persons in a closed community and subsequent cross infection. To investigate this

Received 31 Dec. 1987; accepted 19 April 1988. possibility and to understand the epidemiology of $B$. fragilis, a serogrouping scheme was used to study the relationship among strains isolated from different sites of hospitalised patients and to establish whether or not these strains are different from those isolated in the community.

\section{Materials and methods}

\section{Collection and transport of specimens}

This study was done at Mubarak Al-Kabeer Hospital, Kuwait, between Sep. 1981 and Mar. 1987. Specimens to be examined for $B$. fragilis were collected from patients with wound infections, septicaemia, pelvic infections, infections of the biliary tract, respiratory-tract infections, and deep-seated abscesses. The specimens were wound swabs, blood, endocervical swabs, bile, transtracheal aspirates and exudate from deep-seated abscesses. Rectal swabs were taken from 207 uninfected patients hospitalised for $\geqslant 10$ days and from 232 normal subjects in the community. All specimens were sent to the laboratory in anaerobic transport media (Portagerm 41994 and 41995 ; bioMerieux, Charbonier-le-Bains, France) within 12 h of collection.

\section{Microbiological methods}

Specimens from patients were inoculated on to plates of Blood Agar (CM 55; Oxoid), Schaedler Blood Agar 
(CM 437; Oxoid) with Kanamycin-Vancomycin mixture (55681; bioMerieux) and into Brain Heart Infusion (BHI) Broth (CM 255; Oxoid). Rectal swabs were inoculated only on to Schaedler blood agar containing kanamycin and vancomycin. Cultures were incubated for $48 \mathrm{~h}$ in an anaerobic system (1028; Forma Scientific, Marietta, OH, USA) with an atmosphere of $\mathrm{N}_{2} 90 \%, \mathrm{H}_{2} 5 \%, \mathrm{CO}_{2} 5 \%$. After incubation, BHI-enrichment cultures were subcultured to the above solid media.

\section{Identification of Bacteroides spp.}

Ten colonies, selected from each Schaedler blood-agar plate, were identified according to: colonial appearance; cell morphology; tolerance to bile and brilliant green; resistance to kanamycin (1000 $\mu \mathrm{g}$ disk), penicillin (2 $\mathrm{U})$, colistin $(10 \mu \mathrm{g})$, erythromycin $(60 \mu \mathrm{g})$, rifampicin $(15 \mu \mathrm{g})$ (57311-57371; Anaerodiscs; bioMerieux) and metronidazole (5 $\mu \mathrm{g})$ (195 B; Oxoid); and by the end-products of glucose metabolism as determined by gas-liquid chromatography (Shimatzu Gas Chromatograph GC90; Shimatzu Corporation, Kyoto, Japan). They were also identified with the API 20A system (Holdeman et al., 1977).

Strains of $B$. fragilis were identified as gram-negative anaerobic bacilli, that formed non-pigmented, nonpitting colonies on blood agar, produced acetic and succinic acids as major products of glucose metabolism, were resistant to penicillin, kanamycin and colistin, sensitive to metronidazole and rifampicin, tolerant to bile, and inhibited by brilliant green. They fermented glucose, lactose and sucrose, hydrolysed aesculin, and did not produce indole (Duerden et al., 1980).

\section{Preparation of bacterial suspensions for immunofluorescence}

Suspensions of all strains of $B$. fragilis were prepared for immunofluorescence as described by Elhag et al. (1987).

\section{Preparation of antisera}

Live cultures of strains of Bacteroides from 20 different serotypes (Elhag and Tabaqchali, 1978a; Elhag et al., 1987) were used for the production of antisera. The species, serotypes and sources of the 20 strains of Bacteroides used are shown in table I. Antisera were raised in New Zealand White rabbits, as described by Elhag and Tabaqchali (1978a).

\section{Testing of antisera}

Antisera were tested against 20 strains of Bacteroides of different serotypes by the immunofluorescence method described by Wulff and Lange (1975) with fluorescein isothiocyanate (FITC) conjugated with goat anti-rabbit IgG (74561; Institut Pasteur Production, Marnes-laCoquette, France). Each serum reacted with the homologous strain, but cross reactions also occurred. Crossreacting antibodies were successfully removed from most

Table I. Serotypes and sources of strains of the fragilis group of Bacteroides used for production of antisera

\begin{tabular}{rll}
\hline Serotype & \multicolumn{1}{c}{ Species } & \multicolumn{1}{c}{ Source } \\
\hline 1 & B. thetaiotaomicron & NCTC 10582 \\
2 & B. vulgatus & NCTC 10583 \\
3 & B. fragilis & NCTC 8560 \\
4 & B. fragilis & ATCC 8483 \\
5 & B. ovatus & ATCC 8503 \\
6 & B. distasonis & Cholecystectomy wound \\
7 & B. fragilis & Appendicectomy wound \\
8 & B. fragilis & Peritonitis exudate \\
9 & B. fragilis & Faeces \\
10 & B. distasonis & Faeces \\
11 & B. distasonis & Appendicectomy wound \\
12 & B. fragilis & Cholecystectomy wound \\
13 & B. fragilis & Blood \\
14 & B. fragilis & Blood \\
15 & B. fragilis & Peritonitis exudate \\
16 & B. fragilis & Appendicectomy wound \\
17 & B. fragilis & Appendicectomy wound \\
18 & B. distasonis & Appendicectomy wound \\
19 & B. fragilis & Otitis-media exudate \\
20 & B. fragilis & $\mathrm{MH}$ \\
& & $\mathrm{TH}$ \\
\hline
\end{tabular}

NCTC $=$ National Collection of Type Cultures, London; ATCC $=$ American Type Culture Collection; $\mathrm{BH}=\mathrm{St}$ Bartholomew's Hospital, London; $\mathrm{TH}=\mathrm{St}$ Thomas's Hospital, London; MH = Mubarak Al-Kabeer Hospital, Kuwait. 
sera by absorption with heterologous strains; but some continued to give low-titre cross-reactions with a few strains. These reactions, however, were absent in tests made with serum diluted 1 in 80 . Accordingly, sera used in fluorescence studies were routinely tested at dilutions of 1 in 80 .

\section{Serotyping of strains}

Each antiserum was tested at least three times against all strains of $B$. fragilis as described above. Each of the 20 strains of Bacteroides from the 20 different serotypes was tested in control experiments with homologous antiserum. To suppress non-specific fluorescence, slides were counterstained with Evans's Blue (22274; George T. Gurr Ltd, London) $0.2 \% \mathrm{w} / \mathrm{v}$ in PBS and examined with a fluorescence microscope (Leitz Ortholux II, Germany) with a mercury lamp (HBO 50) as light source. A test was considered positive if, on three occasions, it showed fluorescence equal to, or greater than, that of a control. When serologically identical strains were detected in one specimen, only one was considered. Thus, 343 isolates of $\boldsymbol{B}$. fragilis were selected for serogrouping according to the above criteria; 112 were isolates from infected sites of hospitalised patients and 102 from infected out-patients, 47 from the faeces of uninfected hospitalised patients and 82 from the faeces of healthy subjects in the community.

\section{Statistical methods}

Data were analysed by computer with the SPSSX statistical package for the computation of proximities and the construction of serogroups (Norusis, 1985). Twenty binary variables were defined to denote the presence or absence of the 20 bacteroides antigens among the strains. Similarity between strains could not be measured by the usual euclidian distances because the variables measured were all discrete. The proximities between the strains were measured, therefore, by the Jaccard similarity measure, in which the similarity ratio was in the range $0-1$ and the higher the ratio, the greater the similarity between strains. Clusters (serogroups) were formed by the single-linkage method, whereby each strain was at first considered to be a separate serogroup and then strains with greatest similarities were grouped. At each stage, either new serogroups were formed or the existing ones enlarged so that ultimately all strains formed one group. Thus, strains within serogroups established at the early stages showed greatest similarity, whilst those formed last were least similar. The $\chi^{2}$ test was used to test differences between two independent proportions.

\section{Results}

Reactions with our strains were most frequently obtained with antisera raised against $\boldsymbol{B}$. fragilis, whereas reactions with sera raised against $B$. ovatus, $B$. thetaiotaomicron, $B$. distasonis and $B$. vulgatus were uncommon. The frequency of reactions of the various bacteroides antisera is illustrated in fig 1 . The most frequently reacting antisera (AS) were: AS19 (99), followed by AS20 (68), AS3 (63), AS14 (63) and AS12 (62) and the least frequently reacting were AS1 (6), AS2 (9), AS5 (2), AS6 (4), AS7 (5), AS11 (3) and AS17 (2).

In all, 271 strains $(79 \%)$ were typable. Each of 86 strains reacted with one antiserum and the remaining 185 showed multiple reactions. First, each set of serologically identical strains, with a Jaccard similarity ratio of $1 \cdot 0$, joined to form a serogroup. In these early cluster solutions, however, the number of strains within serogroups was too small to allow a reasonable comparison. We chose for analysis, therefore, the cluster solution showing a reasonable number of strains within serogroups without losing much of the similarity between the strains. At lower cluster solutions, the number of strains within serogroups was high, but the similarity was very low.

The cluster solution selected for analysis, the frequency of occurrence of the serogroups and the bacteroides antigens present in each serogroup are shown in table II. Of the 62 serogroups identified in this cluster solution, 19 had only one bacteroides antigen, whilst the remaining 43 serogroups contained 2-9 antigens. The strains within 24 serogroups were identical (Jaccard similarity ratio $=1$ ) and in 14 the similarity ratio was in the range 0.67 $0 \cdot 75$. The remaining 24 serogroups, however, comprised one strain each.

The distribution of $\boldsymbol{B}$. fragilis serogroups among hospital and community strains is shown in fig. 2 . A greater proportion of hospital strains were typable $(88 \cdot 1 \%)$ than were community strains $(71.2 \%)(p<0.001) ; 89.4 \%$ of faecal strains from uninfected hospitalised patients were typable but only $63.4 \%$ of faecal strains from normal subjects in the community $(\mathrm{p}<0.001)$. There was, however, no significant difference in the typability of strains isolated from infected sites in the hospital and community patients. A greater proportion of isolates from infected sites were typable $(82.7 \%)$ than were faecal isolates $(72.9 \%)(\mathrm{p}<0.05)$.

$B$. fragilis strains of serogroup 2 formed a significantly greater proportion of isolates from the faeces of hospitalised patients $(17 \%)$ than from infected sites (hospital or community) or from faecal specimens from the community $(p<0.01)$. Strains of serogroups $28(\mathrm{p}<0.001)$ and $39(\mathrm{p}<$ 0.01 ) were more prevalent amongst hospital isolates from both faeces and infection sites than among community isolates. On the other hand, strains of serogroups 7, 9 and 25 were evenly distributed between the hospital and the community. No 


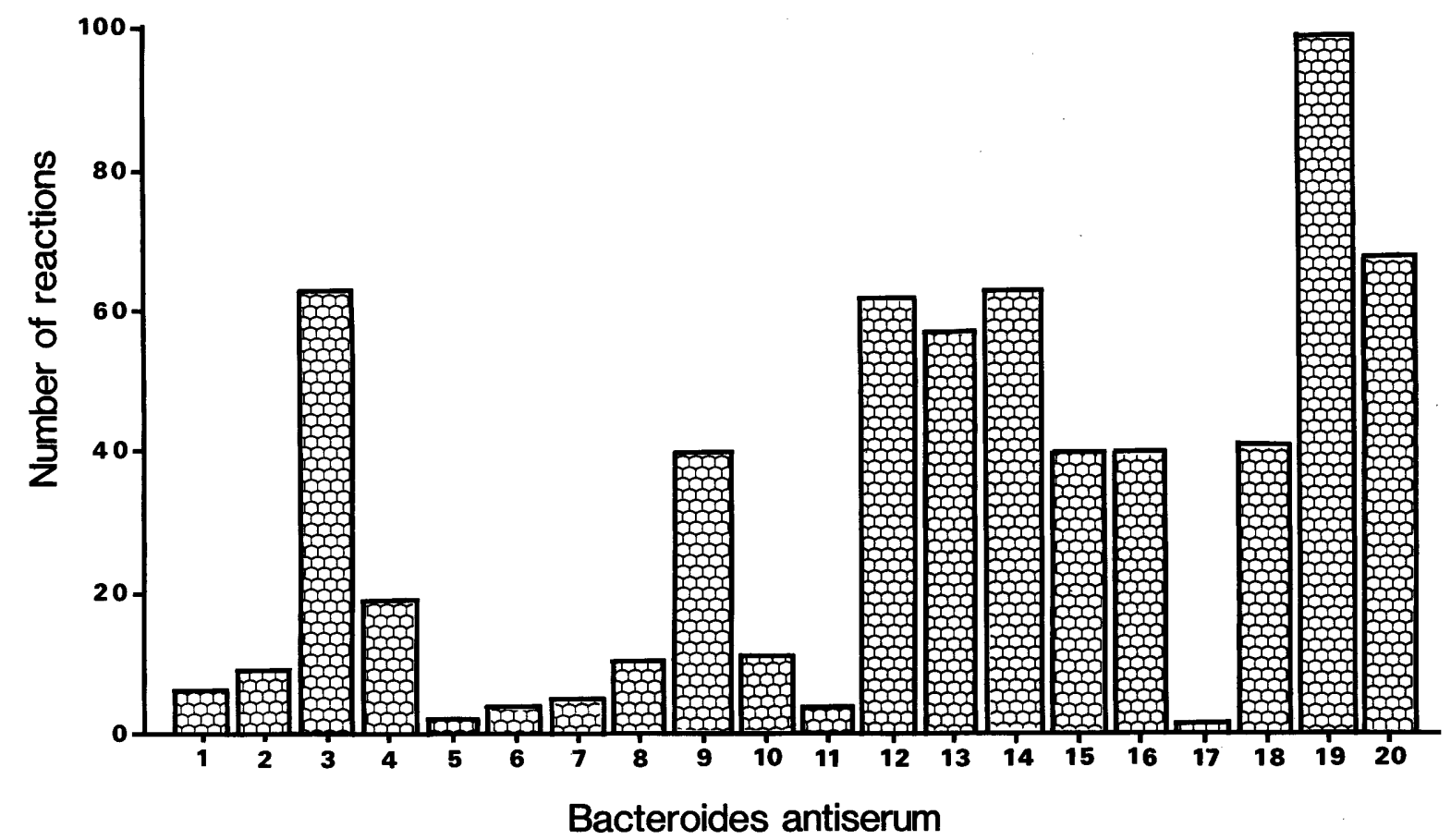

Fig. 1. The frequency of reactions of $B$. fragilis strains with antisera against serotypes $1-20$ of the fragilis group.

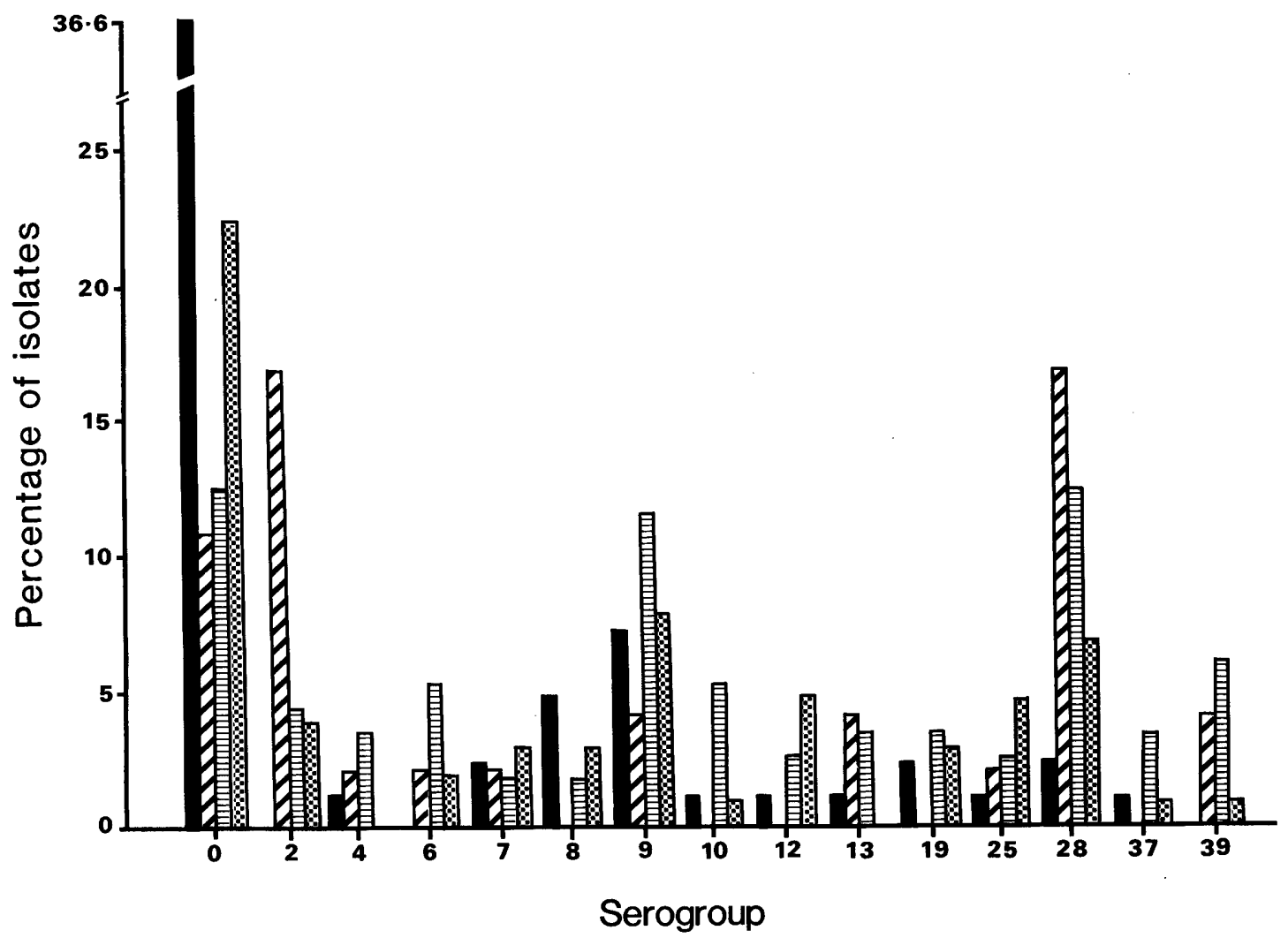

Fig. 2. The distribution of $B$. fragilis serogroups in the community and the hospital: faecal isolates in the community $\square$ and in hospital $\square$; isolates from infected lesions in the community 图 and in hospital 目. 
Table II. B. fragilis serogroups, their range of reactions with Bacteroides antisera and similarity ratio of the strains

\begin{tabular}{|c|c|c|c|c|c|c|c|}
\hline $\begin{array}{l}\text { Sero- } \\
\text { group }\end{array}$ & $\begin{array}{c}\text { Number of } \\
\text { isolates } \\
(n=271)\end{array}$ & Bacteroides antigens & $\begin{array}{c}\text { Similarity } \\
\text { ratio }\end{array}$ & $\begin{array}{l}\text { Sero- } \\
\text { group }\end{array}$ & $\begin{array}{c}\text { Number of } \\
\text { isolates } \\
(n=271)\end{array}$ & Bacteroides antigens & $\begin{array}{l}\text { Similarity } \\
\text { ratio }\end{array}$ \\
\hline 1 & 1 & $13,14,15$ & $\ldots$ & 32 & 2 & 11 & $1 \cdot 0$ \\
\hline 2 & 17 & $9,12,13,14,15,18,19,20$ & $0 \cdot 67$ & 33 & 2 & $8,9,18$ & 0.67 \\
\hline 3 & 1 & $3,9,15,18,19$ & $\cdots$ & 34 & 5 & 12 & $1 \cdot 0$ \\
\hline 4 & 6 & 18,19 & $1 \cdot 0$ & 35 & 5 & $4,9,10,19,20$ & 0.75 \\
\hline 5 & 4 & $3,7,9,14,19$ & $0 \cdot 75$ & 36 & 3 & $8,14,15,16$ & 0.67 \\
\hline 6 & 9 & 19 & $1 \cdot 0$ & 37 & 6 & $13,14,19$ & $1 \cdot 0$ \\
\hline 7 & 8 & 10 & $1 \cdot 0$ & 38 & 3 & 19,20 & $1 \cdot 0$ \\
\hline 8 & 9 & 20 & $1 \cdot 0$ & 39 & 10 & 14,19 & $1 \cdot 0$ \\
\hline 9 & 29 & $2,3,12,13,15,16,18,19,20$ & 0.67 & 40 & 4 & $1,3,12$ & 0.67 \\
\hline 10 & 8 & $4,12,14,18,19$ & 0.67 & 41 & 5 & 3 & $1 \cdot 0$ \\
\hline 11 & 4 & 2 & $1 \cdot 0$ & 42 & 1 & 15,20 & $\ldots$ \\
\hline 12 & 9 & $3,4,6,10,14,15,18,19,20$ & 0.67 & 43 & 1 & 4,9 & $\ldots$ \\
\hline 13 & 7 & 16 & $1 \cdot 0$ & 44 & 2 & 7 & $1 \cdot 0$ \\
\hline 14 & 2 & 12,16 & $1 \cdot 0$ & 45 & 1 & $5,11,13,18,19$ & $\ldots$ \\
\hline 15 & 2 & $13,14,18$ & $0 \cdot 67$ & 46 & 1 & $7,15,16,18,19$ & $\ldots$ \\
\hline 16 & 3 & 8 & 1.0 & 47 & 1 & $7,13,15,16$ & $\ldots$ \\
\hline 17 & 3 & 3,19 & $1 \cdot 0$ & 48 & 1 & $12,14,15,19$ & $\ldots$ \\
\hline 18 & 3 & 12,19 & 1.0 & 49 & 1 & 17 & $\ldots$ \\
\hline 19 & 9 & 9 & $1 \cdot 0$ & 50 & 1 & $2,11,20$ & $\ldots$ \\
\hline 20 & 1 & 1,18 & $\ldots$ & 51 & 1 & 5 & $\ldots$ \\
\hline 21 & 2 & 15,18 & $1 \cdot 0$ & 52 & 1 & $9,15,20$ & $\cdots$ \\
\hline 22 & 1 & 2,12 & $\ldots$ & 53 & 1 & $8,12,13,16,20$ & $\ldots$ \\
\hline 23 & 1 & 6 & $\ldots$ & 54 & 2 & $4,16,20$ & 0.67 \\
\hline 24 & 4 & 1 & $1 \cdot 0$ & 55 & 1 & 4 & $\ldots$ \\
\hline 25 & 10 & 13 & $1 \cdot 0$ & 56 & 1 & $2,13,16$ & $\ldots$ \\
\hline 26 & 2 & 12,20 & $1 \cdot 0$ & 57 & 1 & 6,20 & $\ldots$ \\
\hline 27 & 5 & $2,13,14$ & $0 \cdot 67$ & 58 & 1 & 15 & $\ldots$ \\
\hline 28 & 31 & $3,12,13,14,15,16,19,20$ & 0.67 & 59 & 1 & $12,13,14,16$ & $\ldots$ \\
\hline 29 & 4 & 14 & $1 \cdot 0$ & 60 & 1 & 6,14 & $\ldots$ \\
\hline 30 & 4 & $3,9,12,14,19$ & 0.67 & 61 & 1 & $3,8,15,16$ & $\ldots$ \\
\hline 31 & 4 & 16,19 & $1 \cdot 0$ & 62 & 1 & $12,14,15,17$ & $\cdots$ \\
\hline
\end{tabular}

particular serogroup was dominant among community strains and no difference was found in the distribution of the serogroups between isolates from faeces and those from infected sites. There was no significant association between serogroup and site of infection.

The prevalence of the $B$. fragilis serogroups during the periods $1981-83,1984-85$ and 1986-87 are illustrated in fig. 3 . There was no significant difference in either the typability of $\boldsymbol{B}$. fragilis strains or the prevalence of the different serogroups in relation to time, except that serogroup 28 accounted for $4 \%$ of the isolates in 1981-83 and increased to $13 \%$ in $1986-87(\mathrm{p}<0.05)$.

The distribution of bacteroides serogroups shown in this cluster solution was maintained at lower cluster solutions.

\section{Discussion}

In this study, a serogrouping scheme based on an earlier serotyping method (Elhag and Tabaqchali,
$1978 a$ ) was used to study the epidemiology of $B$. fragilis. When strains of $B$. fragilis were tested against absorbed antisera, some reacted with only one serum but most strains showed multiple reactions. Similar findings have been reported for agglutination reactions (Lambe and Moroz, 1976; Elhag and Tabaqchali, 1978b). We have, therefore, followed an approach similar to that of Lambe and Moroz (1976) to serogroup strains according to the serological patterns obtained. Indeed, with the complexity of $B$. fragilis surface antigens and the wide variety of serological reactions of the fragilis group, such a scheme would be the most appropriate for serogrouping these organisms.

It seems that the antigenic structure of the fragilis group of Bacteroides does not correlate with the species, because strains of $\boldsymbol{B}$. fragilis reacted with sera raised against other species of the fragilis group. These findings are consistent with observations from our earlier study (Elhag and Tabaqchali, $1978 b$ ). The sera showing most frequent reactions were raised against strains of $\boldsymbol{B}$. fragilis isolated 


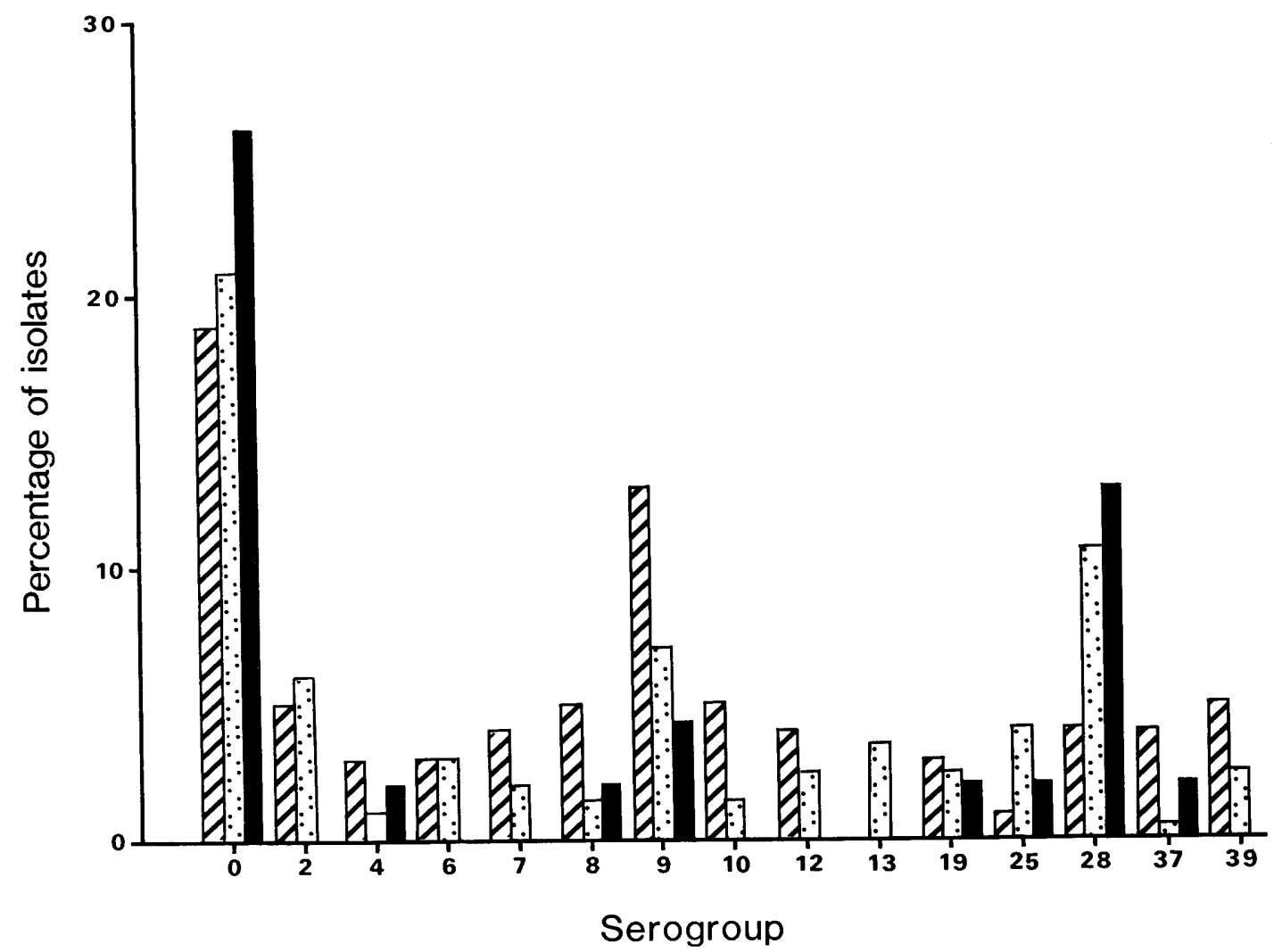

Fig. 3. The frequency of $B$. fragilis serogroups with time; $\mathbb{V} 1981-1983 ;$ : 1984-1985; $1986-1987$.

both in Kuwait and Britain. In a previous study, antisera 12,13 and 14 were among those commonly reacting with British isolates (Elhag and Tabaqchali, 1978b). Although it is known that $B$. fragilis serotypes show a geographical distribution and that certain strains are prevalent in certain areas (Elhag and Tabaqchali, 1978b; Elhag et al., 1987), it appears from our findings that other serotypes are widely spread. These universal strains may prove to be useful as additional antigens for serodiagnosis of B. fragilis infection (Elhag et al., 1987).

Of our $B$. fragilis strains, $79 \%$ were typable, a figure considerably higher than that of British isolates $(62 \%)$ (Elhag and Tabaqchali, 1978b). One possible reason for this difference is that the moresensitive immunofluorescence technique was used to type isolates from Kuwait. The difference may also reflect a variation in the typability of the different species of the fragilis group because, in this study, strains of $B$. fragilis only were typed whereas different species of the fragilis group were tested previously.

The community strains were serologically heter- ogeneous with no serogroup dominant. Naturally, this is an open community in which the natural flora is expected to vary widely. The hospital strains were significantly different from community isolates and several serogroups were dominant among hospital isolates. The prevalence among hospital strains of particular serogroups (especially serogroup 39) consisting of identical isolates strongly suggests colonisation of hospitalised patients by new strains of $B$. fragilis and their spread to other patients in the hospital. It has been shown that gram-negative aerobic bacteria such as Klebsiella spp. are acquired from hospital food, medicaments, and by contact with other patients and staff (Casewell and Phillips, 1978; Maki, 1978). Colonisation by these organisms plays an important role in hospital cross-infection. The mode of hospital spread of $\boldsymbol{B}$. fragilis, however, is unclear at present, but the possibility of spread by routes similar to those of gram-negative aerobes cannot be excluded. $B$. fragilis probably colonises the bowel and other mucous surfaces first and initiates infection thereafter. 
Isolates from infection sites were significantly more typable than faecal isolates. We found, however, no significant difference between serogroups of isolates from infected sites and faecal isolates and no particular serogroup could be associated with infection, a finding contradicting that of our earlier studies (Elhag and Tabaqchali, $1978 b$ ), in which we examined isolates from infected sites only. Had we also examined faecal strains there might have been no difference. Over the 6year period of study, there was a significant increase in the incidence of one serogroup, indicating a tendency towards seasonal variation of $B$. fragilis

\section{REFERENCES}

Casewell M, Phillips I 1978 Food as a source of Klebsiella species for colonisation and infection of intensive care patients. Journal of Clinical Pathology 31 : 845-849.

Duerden B I, Collee J G, Brown R, Deacon A G, Holbrook W P 1980 A scheme for the identification of clinical isolates of gram-negative anaerobic bacilli by conventional bacteriological tests. Journal of Medical Microbiology 13: 231245.

Elhag K M, Tabaqchali S $1978 a$ A study of the surface and somatic antigens of Bacteroides fragilis. Journal of Hygiene 80: $439-449$.

Elhag K M, Tabaqchali S $1978 b$ The distribution of Bacteroides fragilis serotypes amongst clinical strains. Journal of Hygiene 81: 89-97.

Elhag K M, Bahar A M, Mahajan K K 1987 Difficulties in the serodiagnosis of infection with the fragilis group of Bacteroides. Journal of Medical Microbiology 23 : 125-131.

Elhag K M, Bettelheim K A, Tabaqchali S 1977 Serological studies of Bacteroides fragilis. Journal of Hygiene 79: 233241.

Elhag K M, Bahar A M, Alwan M H, Mahajan K K, Sherif R $A$, Senthilselvan A The role of anaerobes in surgical infections in Kuwait: a multi-centre study of 379 patients. Medical Principles and Practice in press.

Gorbach S L, Bartlett J G 1974 Anaerobic infections (first of three parts). New England Journal of Medicine 290: 11771184.

Holdeman L V, Cato E P, Moore W E C (eds) 1977 Anaerobe laboratory manual, 4 th edn. Anaerobe Laboratory, Virginia Polytechnic Institute and State University, Blacksburg, VA.

Jones G R, Gemmell C G 1986 Effects of Bacteroides asaccharolyticus cells and Bacteroides fragilis surface components on serum opsonisation and phagocytosis. Journal of Medical Microbiology 22: 225-229.

Kasper D L, Onderdonk A B 1982 Infection with Bacteroides serogroups. This appears, however, to take place over a long period.

We conclude, therefore, that $B$. fragilis can colonise hospitalised patients and spread to other patients in the hospital. On the basis of these findings, we may consider $B$. fragilis to be a hospital pathogen that plays a role in hospital crossinfection. Nevertheless, further knowledge of its sources and modes of spread is required.

This work was supported by Kuwait University Research Council (grant no. MI 007). We thank Mrs Aleyamma Alex for technical assistance and Mr Mohamed Sidiq for the drawings.

fragilis: pathogenesis and immunoprophylaxis in an animal model. Scandinavian Journal of Infectious Diseases, Suppl 31 : $28-33$.

Lambe D W, Moroz D A 1976 Serogrouping of Bacteroides fragilis subspecies fragilis by the agglutination test. Journal of Clinical Microbiology 3: 586-592.

Maki D 1978 Control of colonization and transmission of pathogenic bacteria in the hospital. Annals of Internal Medicine 89: 777-780.

Moore W E C 1977 Anaerobes as normal flora: gastrointestinal tract. In: Finegold S M (ed) Metronidazole: proceedings of the international metronidazole conference. (Excerpta Medica International Congress Series No. 438) Excerpta Medica, Amsterdam pp. 222-228.

Moore W E C, Cato E P, Holdeman L V 1969 Anaerobic bacteria of the gastrointestinal flora and their occurrence in clinical infections. Journal of Infectious Diseases 119: 641649.

Namavar F, Verweij-Van Vught A M J J, Bal M, MacLaren D M 1987 Effect of Bacteroides fragilis cellular components on chemotactic activity of polymorponuclear leukocytes towards Escherichia coli. Journal of Medical Microbiology 24: 119-124.

Norusis M J 1985 Cluster analysis; proximities. In: SPSSX: Advanced statistics guide. McGraw-Hill Company, New York, pp 165-191 and 462-467.

Tabaqchali S, Fiddian P A, Elhag K, Kasper D L 1982 Capsular and " $O$ " serotype determinants of Bacteroides fragilis. Infection 10: 333-337.

Romond Ch, Beerens H, Wattre P 1972 Identification sérologiques des bactérides en relation avec leur pouvoir pathogène. (Serological identification of bacteroides connected with their pathogenicity). Archives Roumaines de Pathologie Experimentale et de Microbiologie 31 : 351-355.

Wulff H, Lange J V 1975 Indirect immunofluorescence for the diagnosis of Lassa fever infection. Bulletin of the World Health Organization 52: 429-436. 